\title{
Solid-State NMR Reveals Structural Differences between Fibrils of Wild-Type and Disease-Related A53T Mutant $\alpha$-Synuclein
}

\author{
Henrike Heise $^{1 \star}$, M. Soledad Celej ${ }^{2}$, Stefan Becker ${ }^{1}$, Dietmar Riedel ${ }^{3}$, \\ Avishay Pelah ${ }^{2}$, Ashutosh Kumar ${ }^{1}$, Thomas M. Jovin ${ }^{2}$ \\ and Marc Baldus ${ }^{1 *}$
}

${ }^{1}$ Department of NMR-Based Structural Biology, Max Planck Institute for Biophysical Chemistry, Solid-State NMR Group, Am Fassberg 11, D-37077 Göttingen, Germany

${ }^{2}$ Laboratory of Cellular Dynamics, Max Planck Institute for Biophysical Chemistry, Am Fassberg 11, D-37077 Göttingen, Germany

${ }^{3}$ Department for Electron Microscopy, Max Planck Institute for Biophysical Chemistry, Am Fassberg 11, D-37077 Göttingen, Germany

Received 4 March 2008; received in revised form 7 May 2008;

accepted 13 May 2008

Available online 17 May 2008

Edited by A. G. Palmer III

\begin{abstract}
Fibrils from the Parkinson's-disease-related A53T mutant of $\alpha$-synuclein were investigated by solid-state NMR spectroscopy, electron microscopy, and atomic force microscopy. Sequential solid-state NMR resonance assignments were obtained for a large fraction of the fibril core. Experiments conducted above and below the freezing point suggest that the fibrils contain regions with increased mobility and structural elements different from $\beta$ strand character, in addition to the rigid $\beta$-sheet-rich core region. As in earlier studies on wild-type $\alpha$-synuclein, the C-terminus was found to be flexible and unfolded, whereas the main core region was highly rigid and rich in $\beta$-sheets. Compared to fibrils from wild-type $\alpha$-synuclein, the wellordered $\beta$-sheet region extends to at least L38 and L100. These results demonstrate that a disease-related mutant of $\alpha$-synuclein differs in both aggregation kinetics and fibril structure.
\end{abstract}

(C) 2008 Elsevier Ltd. All rights reserved.
Parkinson's disease, the most common neurodegenerative movement disorder, is caused by loss of dopaminergic neurons in the substantia nigra, accompanied by formation of cytosolic filamentous inclu-

${ }^{*}$ Corresponding authors. E-mail addresses: h.heise@fz-juelich.de; maba@mpibpc.mpg.de.

Present address: Henrike Heise, Institute for Physical Biology, University Düsseldorf, D-40225 Düsseldorf, Germany, and Forschungszentrum Jülich, INB-2, D-52425 Jülich, Germany.

Abbreviations used: AFM, atomic force microscopy; EM, electron microscopy; MAS, magic angle spinning; ssNMR, solid-state NMR; HETCOR, heteronuclear correlation. sions called Lewy bodies. ${ }^{1}$ The major constituent of these inclusions consists of fibrillar $\alpha$-synuclein, ${ }^{2}$ a $140-$ residue protein that, in its native conformation, is largely unfolded. The central role of $\alpha$-synuclein aggregation in the etiology of Parkinson's disease has been further corroborated by the identification of a locus triplication of the gene encoding $\alpha$-synuclein, ${ }^{3}$ as well as the three point mutations $\mathrm{A} 53 \mathrm{~T},{ }^{4,5} \mathrm{~A} 30 \mathrm{P}^{6},{ }^{6}$ and $\mathrm{E} 46 \mathrm{~K}^{7}{ }^{7}$ as cause of autosomal dominant Parkinson's disease. While dopaminergic nerve cell loss clearly is linked to enhanced $\alpha$-synuclein aggregation, it is still unclear whether mature amyloid fibrils or oligomeric intermediates are the toxic agents that lead to cell death. ${ }^{8}$

Since their discovery, the three disease-related mutants have been the subject of intense study, as 
differences in aggregation behavior and morphology with respect to the wild-type form may yield valuable insights into the fundamental processes underlying amyloidogenic diseases. ${ }^{9-21}$ As in the case of wild-type $\alpha$-synuclein, all three mutants are natively unfolded, ${ }^{15}$ although the propensities for residual secondary structure around the mutation sites are slightly altered. ${ }^{10,14,20}$ In comparison with the wildtype form, significant acceleration of fibrillization has been observed for the mutants $A 53 \mathrm{~T}^{9,10,15,17}$ and E46K, ${ }^{16,18,20}$ whereas contradicting observations regarding oligomerization and aggregation kinetics have been reported for the A30P mutant. ${ }^{10,11,15}$ The morphology of in vitro aggregates of wild-type $\alpha$ synuclein and its familial mutants has also been studied intensively by atomic force microscopy (AFM) and electron microscopy (EM). 12,16,19 Whereas fibrils of the mutant form A30P have an appearance similar to that of wild-type $\alpha$-synuclein, fibrils from the mutants A53T and E46K are more twisted. However, the overall morphology of amyloid fibrils, in general, depends strongly on fibrillization conditions, including $\mathrm{pH}$, temperature, salt concentration, and agitation of the solution. ${ }^{22}$ Upon fibrillization of wild-type $\alpha$-synuclein at physiological temperature and $\mathrm{pH}$, at least two different polymorphic forms-one consisting of straight fibrils and the other consisting of irregularly twisted fibrils-have been obtained and characterized. ${ }^{23}$

A more detailed picture of the molecular arrangement within the fibril can be obtained by solid-state magic angle spinning (MAS) NMR spectroscopy, which has emerged as a powerful tool for structure elucidation in amyloid fibrils (for recent reviews, see, e.g., Tycko ${ }^{24}$ and Heise ${ }^{25}$ and references cited therein). Studies of wild-type $\alpha$-synuclein have revealed a $\beta$-sheet-rich core region spanning residues from at least 38 to 94 for the two different polymorphic isoforms, whereas the C-terminus was flexible and unfolded in both cases. ${ }^{23}$ Our structural findings were largely confirmed by other solid-state NMR (ssNMR) data reported recently, ${ }^{26,27}$ although significant spectral changes that could indicate the existence of a yet third isoform were apparent. While ssNMR spectra of fibrils from the A30P mutant exhibit differences with respect to wildtype $\alpha$-synuclein, ${ }^{28}$ concrete structural insights into the effects of protein mutation on fibril formation are as yet very limited. In the following, we report studies on fibrils grown from the disease-related A53T mutant of $\alpha$-synuclein by ssNMR, EM, and AFM.

\section{Sample preparation and characterization by EM and AFM}

The A53T mutant of $\alpha$-synuclein was expressed recombinantly in Escherichia coli cultures as described before. ${ }^{23}$ The protein was labeled uniformly with ${ }^{13} \mathrm{C}$ and ${ }^{15} \mathrm{~N}$ isotopes in all amino acid residues except lysine - a condition achieved by using minimal medium containing ${ }^{13} \mathrm{C}_{6}$-glucose, ${ }^{15} \mathrm{~N}$ ammonium chloride, and $100 \mathrm{mg} / \mathrm{l}$ non-isotope-labeled lysine for expression.

Fibrils were prepared from a solution with a protein concentration of $\sim 250 \mu \mathrm{M}$ in $25 \mathrm{mM}$ Tris$\mathrm{HCl}$ buffer $\left(\mathrm{pH} 7.5\right.$, at $25{ }^{\circ} \mathrm{C}$ ) and $0.02 \% \mathrm{NaN}_{3}$. Fibrillization was achieved by incubation at $37{ }^{\circ} \mathrm{C}$ for 7 days, as described before. ${ }^{23}$ The time course of aggregation was monitored by standard thioflavin $\mathrm{T}$ fluorescence assay (Supplementary Information, Fig. SI1). ${ }^{29}$ Fibrils were isolated by three consecutive cycles of centrifugation $\left(10^{5} \mathrm{~g}\right)$ and resuspension in Tris- $\mathrm{HCl}$ buffer.

In Fig. 1, EM and AFM pictures of fibrils grown from the full-length A53T mutant of $\alpha$-synuclein are shown. The sample displayed long fibrils with a diameter of $160 \AA$, presumably composed of two protofilaments with a diameter of $80 \AA$. The majority of the fibrils had a straight appearance.

\section{ssNMR spectra and resonance assignments}

Sequential resonance assignments for A53T $\alpha-$ synuclein were made based on interresidue crosscorrelations in two-dimensional spin diffusion spectra recorded under weak coupling conditions ${ }^{30}$ and from comparison of heteronuclear nitrogencarbon correlation spectra (NCACX and NCOCX) (Supplementary Information, Fig. SI2). ${ }^{31}$ Unambiguous sequential resonance assignments from

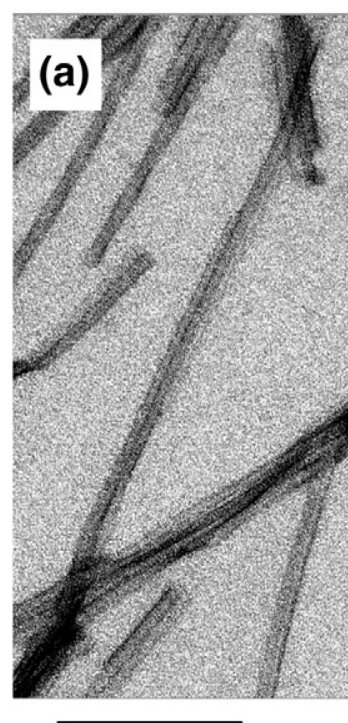

$200 \mathrm{~nm}$

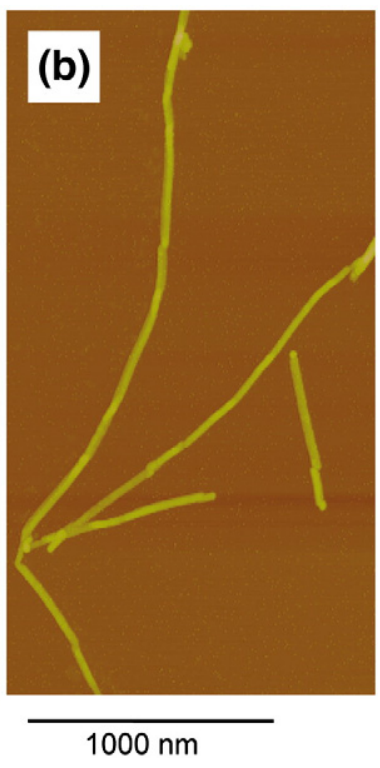

$1000 \mathrm{~nm}$
Fig. 1. EM (a) and AFM (b) images of A53T $\alpha$-synuclein fibrillized at $37^{\circ} \mathrm{C}$. For EM measurements, amyloid fibrils, which were resuspended from the pellet, were prepared on a glow-discharged carbon foil and stained with 1\% uranyl acetate. The sample was evaluated with a CM 120 Phillips TEM. Pictures were taken with a $2048 \times 2048$ Tietz TemCam 224A camera in spot mode at a magnification of $195,000 \times$ at $-1.15 \mu \mathrm{m}$ defocus. For the AFM image, a 5- to $10-\mu l$ drop of a dilute aqueous amyloid solution was spincoated to dryness on a freshly cleaved mica surface. A Digital Instruments (Veeco) MultiMode scanning probe microscope IIIla was used. The sample was imaged in air at a 1-Hz scan rate using silicon tips. 
both types of correlation spectra could be obtained for the amino acids from two stretches comprising residues V63-G73 and T81-A90. Furthermore, pairwise sequential assignments were obtained for G36Y39, G41/S42, A56/E57, E61/Q62, A91-F94, and D98-G101 from either homonuclear or heteronuclear correlations (HETCORs) alone (Supplementary Information).

In hydrated amyloid fibrils, different regions of a protein may exhibit diverse residual mobility. ${ }^{23,32}$ Therefore, ssNMR spectra based on dipolar transfer mechanisms often show only a subset of all resonances in the protein. Freezing the sample reduces the overall mobility such that flexible parts may give rise to more or less inhomogeneously broadened signals, depending on the degree of disorder. Alternatively, flexible regions can selectively be detected by applying adequate mobility filters in the MAS NMR experiments. ${ }^{23,25,33,34}$

Figure $2 \mathrm{a}$ shows two ${ }^{13} \mathrm{C} /{ }^{13} \mathrm{C}$ correlation spectra recorded at different temperatures. Sample temperatures under different cooling conditions at different MAS speeds were calibrated externally with an accuracy of $\pm 5{ }^{\circ} \mathrm{C}$ by the temperaturedependent paramagnetic ${ }^{1} \mathrm{H}$ chemical shift of nickelocene, as described previously. ${ }^{35}$ In addition, sample freezing was observed by a drastic change in the probehead ${ }^{1} \mathrm{H}$ tuning around a temperature of $-10{ }^{\circ} \mathrm{C}$ and by a significant line broadening of ${ }^{1} \mathrm{H}$ water resonance. The first spectrum was recorded at $\sim 0{ }^{\circ} \mathrm{C}$ (i.e., slightly above the freezing point), while the second spectrum was obtained under similar conditions but at an effective sample temperature of approximately $-20{ }^{\circ} \mathrm{C}$ (i.e., well below the sample (a)

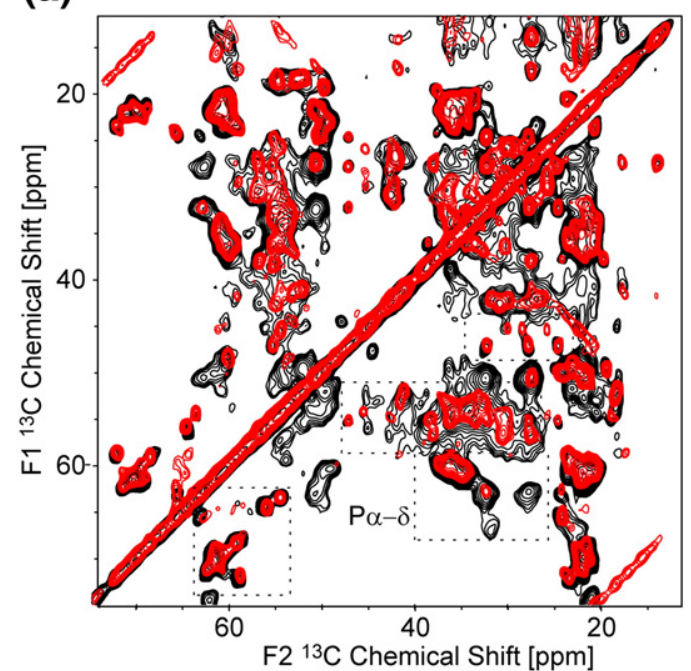

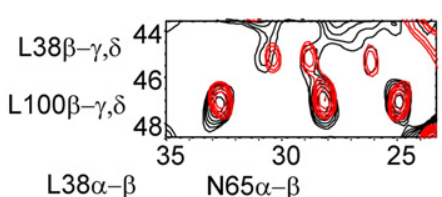
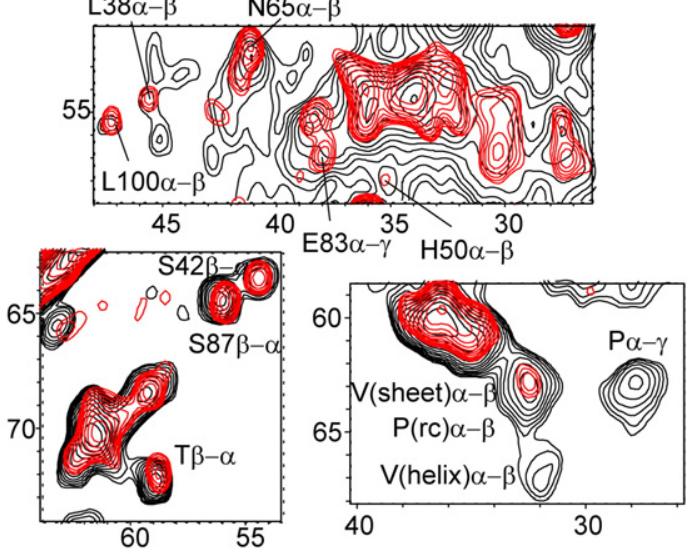

(b)
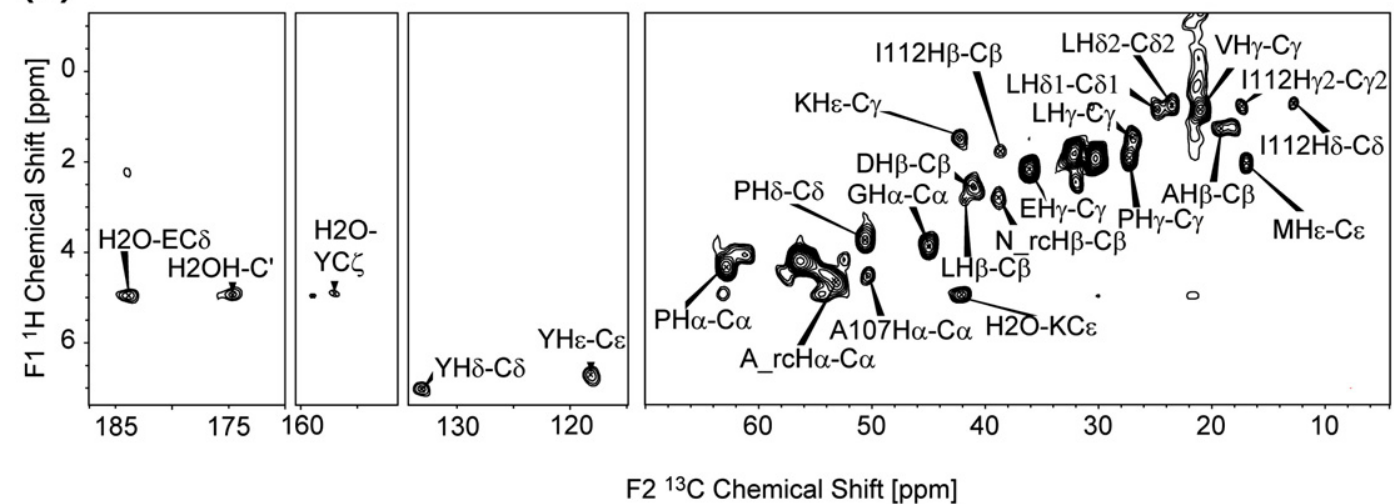

Fig. 2. (a) Two-dimensional ${ }^{13} \mathrm{C} /{ }^{13} \mathrm{C}$ correlation spectra of $\mathrm{A} 53 \mathrm{~T} \alpha$-synuclein fibrils recorded at effective sample temperatures of $\sim 0{ }^{\circ} \mathrm{C}$ (red) and $-20^{\circ} \mathrm{C}$ (black). Homonuclear mixing was achieved by proton-driven spin diffusion for $20 \mathrm{~ms}$ in all cases; the external magnetic field strength was $14 \mathrm{~T}$. MAS frequencies were $8.35 \mathrm{kHz}$ (red) and $9.375 \mathrm{kHz}$ (black), respectively. Spectra were acquired with $320 t_{1}$ increments corresponding to a maximum $t_{1}$ of $5.3 \mathrm{~ms}$, with 128 scans per $t_{1}$ increment. During $t_{1}$ and $t_{2}$, SPINAL-64 decoupling ${ }^{40}$ on protons with field strengths of up to $90 \mathrm{kHz}$ was employed. The duration of the initial cross-polarization pulse was $400 \mu$ s in all cases. (b) Two-dimensional ${ }^{1} \mathrm{H} /{ }^{13} \mathrm{C}^{1} \mathrm{H}-\mathrm{T}_{2}$ filtered HETCOR spectrum of A53T $\alpha$-synuclein. The spectrum was recorded at a magnetic field strength of $9.4 \mathrm{~T}$ with a spinning speed of $9.5 \mathrm{kHz}$, at a sample temperature of $+5{ }^{\circ} \mathrm{C}$. The $\mathrm{T}_{2}$ filter delay was $2 \times 100 \mu \mathrm{s}$, and the contact time was $1 \mathrm{~ms}$. The spectrum was recorded without homonuclear decoupling during $t_{1}$. Eighty $t_{1}$ increments, amounting to a maximum $t_{1}$ time of $8 \mathrm{~ms}$, were recorded with 512 scans per slice. 
freezing point). As in the case of spectra recorded on fibrils of wild-type $\alpha$-synuclein, the signals are wellresolved with line widths of between $0.5 \mathrm{ppm}$ and 1 ppm for ${ }^{13} \mathrm{C}$ and ${ }^{15} \mathrm{~N}$ resonances. All crosscorrelations stemming from serine, threonine, and leucine residues giving rise to resolved signals below the freezing point are also clearly present in the spectrum recorded at $0{ }^{\circ} \mathrm{C}$. This holds also for signals from valine residues with secondary chemical shifts indicative of $\beta$-sheet conformation. However, the region of the $C^{\alpha} / C^{\beta}$ and $C^{\alpha} / C^{\gamma}$ crosspeaks of glutamine and glutamic acid appears significantly more crowded in the spectrum of the frozen sample, which can be either due to homogeneously or inhomogeneously broadened resonances or due to an increased number of resonances. Likewise, cross-correlations typical of proline with random-coil chemical shifts are significantly enhanced in the low-temperature spectrum. Furthermore, at least one valine cross-correlation with secondary chemical shifts typical of $\alpha$-helical conformation is observed in the low-temperature spectrum, but is absent from the high-temperature spectrum. All 5 proline residues and 10 of 18 glutamic acid residues in the sequence are located in the C-terminus, which has already been shown to be mobile and to be not involved in the rigid fibril core in fibrils from wild-type $\alpha$-synuclein ${ }^{23,36-}$ 38 (Heise et al., in preparation).

To further probe the mobility of residues on a molecular level, we recorded a ${ }^{1} \mathrm{H} /{ }^{13} \mathrm{C}$ HETCOR spectrum with a proton $\mathrm{T}_{2}$ filter of $2 \times 100 \mu \mathrm{s}$ and a long cross-polarization step of $1 \mathrm{~ms}$ (Fig. 2b). Under these conditions, only resonances from mobile segments of immobilized proteins were detected. ${ }^{23,25}$
As with wild-type $\alpha$-synuclein, resonances with random-coil chemical shifts could be identified unambiguously for the following amino acid types: alanine, asparagine, aspartate, glycine, glutamic acid, isoleucine, leucine, lysine, methionine, proline, and tyrosine, whereas a ${ }^{13} \mathrm{C}$ resonance of around $70 \mathrm{ppm}$, which would be characteristic of threonine $\mathrm{C}^{\beta}$ in random-coil conformation, is clearly missing from the subsection of the mobile amino acids. One signal set characteristic of alanine preceding a proline residue can be assigned to A107, whereas the mobile isoleucine residue is assigned to I112, as the identity of I88 is unambiguous from the ${ }^{13} \mathrm{C} /{ }^{13} \mathrm{C}$ correlation spectra. A summary of ssNMR resonance assignments is given in Supplementary Information. Signals with secondary chemical shifts deviating by $>1 \mathrm{ppm}$ from the random-coil value cannot be identified in the spectra. In particular, the presence of $\alpha$-helical valine, and of proline and serine in an extended conformation in the highly mobile regions of the protein can be excluded with certainty. As discussed previously for wild-type $\alpha$-synuclein, the distribution of amino acids in the mobile part of A53T $\alpha$-synuclein fibrils speaks in favor of a mobile unstructured C-terminus, whereas the absence of mobile threonine residues indicates that the N-terminus from residue 22 onwards is more rigid, although not necessarily well-structured.

\section{The $\beta$-sheet core of A53T $\alpha$-synuclein fibrils is extended with respect to wild-type $\alpha$-synuclein}

The most striking difference between the fibrils of wild-type $\alpha$-synuclein and the fibrils of A53T

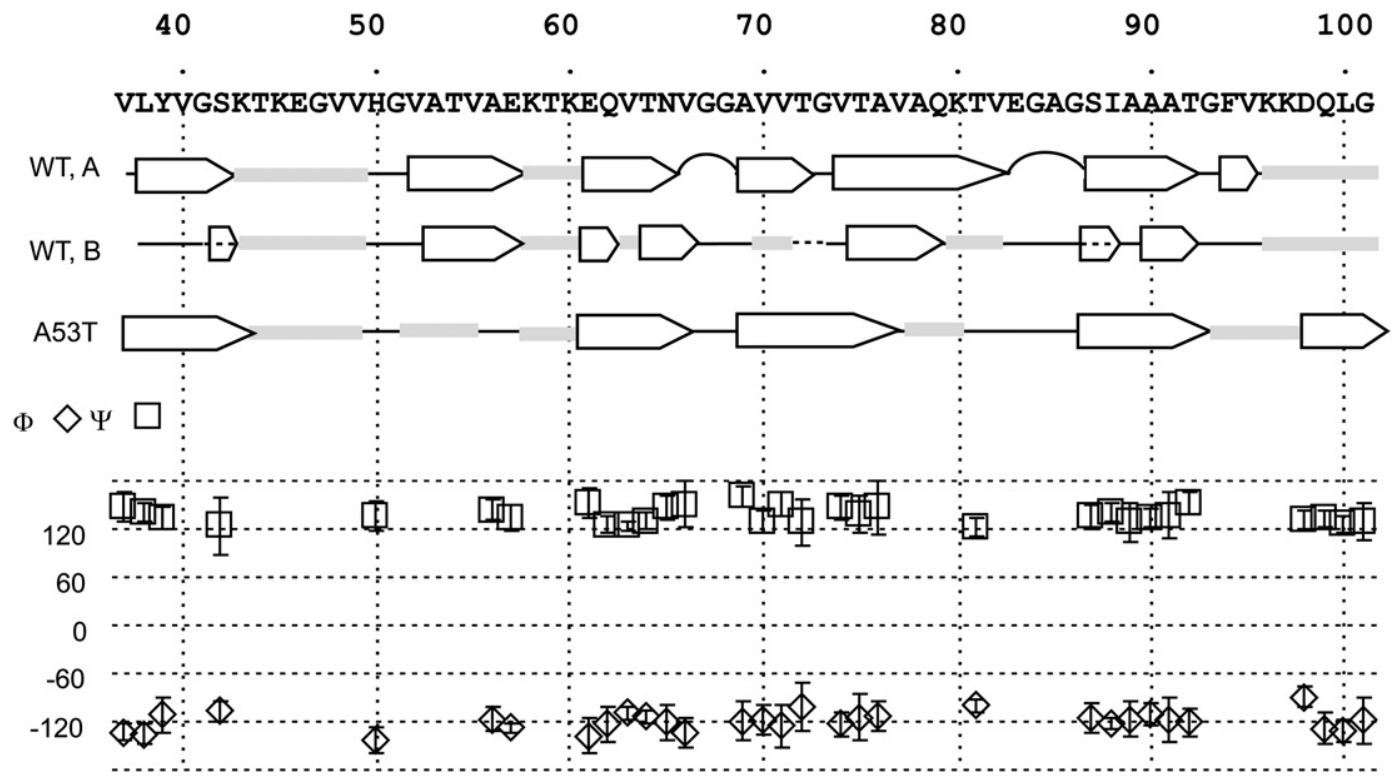

Fig. 3. Backbone angles obtained with TALOS ${ }^{39}$ analysis of the chemical shifts. Only torsion angle pairs where at least eight hits were found are plotted, together with the error bars. For comparison, $\beta$-strands determined earlier for both wild-type forms ${ }^{23}$ are given together with the $\beta$-strands of A53T. Nonassigned amino acids are marked with gray bars, and $\beta$-sheets as determined by TALOS are indicated by white arrows; assigned residues for which TALOS did not yield conclusive results are marked with a black line. 
mutant relates to resonances found at the N- and Cterminal ends of the fibril core. In the mutant form, we detected signal patterns of two different leucine residues with extremely low-field secondary chemical shifts for $C^{\beta}$, typical of $\beta$-sheet conformation. In contrast to ssNMR data of fibrils from wild-type $\alpha$ synuclein, in which correlations with such pronounced $\beta$-sheet characteristic chemical shifts were missing, ${ }^{23,26,27}$ we could unambiguously assign those two leucine residues flanking the core region (i.e., residues L38 and L100). Leucine residues L8 and L113 did not give rise to resolved signals in the dipolar ${ }^{13} \mathrm{C} /{ }^{13} \mathrm{C}$ spectra. These residues most likely contribute to the leucine resonances in the $\mathrm{T}_{2}$ filtered HETCOR spectra displaying mobile parts of A53T $\alpha$-synuclein fibrils. The large secondary chemical shifts of L100 and L38, characteristic of $\beta$-sheet secondary structure, are in contrast to previous results on both wild-type forms, with which only L38 could be assigned with resonances close to random-coil chemical shifts (although a TALOS analysis assigned L38 in A-type fibrils as part of a $\beta$-strand). ${ }^{23}$ Likewise, in the spectra of Kloepper et al., characteristic signal patterns for leucine residues in $\beta$-sheet conformation are clearly missing in all spectra recorded at high and low temperatures ${ }^{26}$ and from dehydrated fibrils, indicating that in these samples, residues L100 and L38 either reside in lesswell-defined regions or exhibit much less pronounced characteristic $\beta$-strand secondary shifts. ${ }^{27}$ An increase in $\beta$-strand character in the -alpha-synuclein mutant is furthermore supported by pairwise assignments for G36, V37 and D98, and Q99 and G101.

In Fig. 3, the results of a TALOS ${ }^{39}$ analysis of the chemical shifts of all assigned residues from V37 to G101 are displayed and compared with those of the $\beta$-sheet regions identified earlier for both forms of wild-type $\alpha$-synuclein. Compared to the latter, the core region of the mutant, characterized by ordered $\beta$-sheets, extends further towards the C-terminus, whereas the main core region is preserved, except for minor differences.

\section{Conclusions}

We have studied fibrils grown from the diseaserelated A53T mutant of $\alpha$-synuclein by solid-state MAS NMR spectroscopy. Fibrils consist of a wellordered rigid core region that is rich in $\beta$-sheets and a highly flexible and unfolded C-terminus, whereas the N-terminus lacks high mobility starting from residue 22. Site-specific resonance assignments for a large part of the amino acids in the core regions were obtained from two-dimensional homonuclear ${ }^{13} \mathrm{C} /{ }^{13} \mathrm{C}$ and heteronuclear ${ }^{15} \mathrm{~N} /{ }^{13} \mathrm{C}$ correlation spectra. Intrinsic dynamics of the protein were probed by spectroscopy at different temperatures, as well as by a combined mobility filter experiment specific for mobile regions of immobilized molecules. According to our results, almost all resonances indicative of a $\beta$-sheet conformation are in rigid protein compartments. Upon freezing of the sample, resonances with random-coil and $\alpha$-helical secondary chemical shifts appear in the spectrum, indicating that the fibrils contain regions with increased mobility and structural elements other than $\beta$-sheet, while the intensities of resonances in $\beta$ strands do not increase unexpectedly. For example, the low-temperature spectrum indicates the existence of at least one valine residue with a chemical shift typical of $\alpha$-helical conformation, which is neither part of the flexible C-terminus nor part of the rigid core region, but belongs to a region of intermediate mobility. These findings agree well with our previous investigations on wild-type fibrils, in which sequential resonance assignments for a large part of the core region could be obtained from spectra recorded above the freezing point. Interestingly, they differ from recent investigations of Kloepper et al., where the resonance intensities of almost all threonine and serine resonances and most alanine resonances, whether in the $\beta$-sheet core region or not, were reduced beyond the detection limit in spectra taken above the freezing point. Such discrepancies can be explained by the existence of different fibril isoforms, characterized by variations in chemical shifts and molecular mobility. Similar to our previous results obtained on wild-type $\alpha$-synuclein, the C-terminus is unfolded and highly dynamic.

The striking difference between the A53T and wild-type $\alpha$-synuclein is an increase in the $\beta$-strand character of two leucine residues at the edges of the core region, indicating an extension of the rigid wellordered $\beta$-sheet-rich core region towards at least residues L38 and L100. Inasmuch as the mutation site is 48 residues removed from L100, a conformational change of this residue in the monomer directly caused by the mutation appears unlikely. Solution NMR studies on spin-labeled monomers have suggested a reduced shielding effect between different domains of the molecule as a cause for the accelerated aggregation kinetics in $\mathrm{A53T}{ }^{21}$ which could result in an earlier or a more efficient exposure of the C-terminal region leading to the more facile association of neighboring molecules. Alternatively, the accelerated aggregation kinetics itself could result in a different pattern of molecular assembly characterized by an extended $\beta$-sheet core region. However, in fibrils of both the mutant and the wild-type $\alpha$-synuclein, the $C$-terminus extending from at least residue 107 is flexible, whereas the $\mathrm{N}$-terminus extending from residue 22 is rigid.

These results provide important insights into the effect of changed aggregation properties on the final fibril morphology of one disease-related mutant of $\alpha$-synuclein. Further studies of this kind may help to delineate the relationship between the etiology of Parkinson's disease and protein misfolding.

\section{Acknowledgements}

H.H. gratefully acknowledges support through a Liebig fellowship of the Fonds der Chemischen Industrie. Karin Giller is thanked for expert 
technical assistance. M.S.C. is the recipient of a postdoctoral fellowship from the Alexander von Humboldt Foundation. This work was conducted within the scientific scope of the Deutsche Forschungsgemeinschaft Center for Molecular Physiology of the Brain and the Graduiertenkolleg GRK 782 in Göttingen, Germany.

\section{Supplementary Data}

Supplementary data associated with this article can be found, in the online version, at doi:10.1016/ j.jmb.2008.05.026

\section{References}

1. Cookson, M. R. (2004). Molecules that cause or prevent Parkinson's disease. PLoS Biol. 2, 1717-1720.

2. Spillantini, M. G., Schmidt, M. L., Lee, V. M. Y., Trojanowski, J. Q., Jakes, R. \& Goedert, M. (1997). $\alpha$-Synuclein in Lewy bodies. Nature, 388, 839-840.

3. Singleton, A. B., Farrer, M., Johnson, J., Singleton, A., Hague, S., Kachergus, J. et al. (2003). $\alpha$-Synuclein locus triplication causes Parkinson's disease. Science, 302, 841.

4. Polymeropoulos, M. H., Higgins, J. J., Golbe, L. I., Johnson, W. G., Ide, S. E., Di Iorio, G. et al. (1996). Mapping of a gene for Parkinson's disease to chromosome 4q21-q23. Science, 274, 1197-1199.

5. Polymeropoulos, M. H., Lavedan, C., Leroy, E., Ide, S. E., Dehejia, A., Dutra, A. et al. (1997). Mutation in the $\alpha$-synuclein gene identified in families with Parkinson's disease. Science, 276, 2045-2047.

6. Krüger, R., Kuhn, W., Müller, T., Woitalla, D., Graeber, M., Kosel, S. et al. (1998). A30P mutation in the gene encoding $\alpha$-synuclein in Parkinson's disease. Nat. Genet. 18, 106-108

7. Zarranz, J. J., Alegre, J., Gomez-Esteban, J. C., Lezcano, E., Ros, R., Ampuero, I. et al. (2004). The new mutation, E46K, of $\alpha$-synuclein causes Parkinson and Lewy body dementia. Ann. Neurol. 55, 164-173.

8. Caughey, B. \& Lansbury, P. T. (2003). Protofibrils, pores, fibrils, and neurodegeneration: separating the responsible protein aggregates from the innocent bystanders. Annu. Rev. Neurosci. 26, 267-298.

9. Conway, K. A., Harper, J. D. \& Lansbury, P. T. (1998). Accelerated in vitro fibril formation by a mutant $\alpha$-synuclein linked to early-onset Parkinson disease. Nat. Med. 4, 1318-1320.

10. Narhi, L., Wood, S. J., Steavenson, S., Jiang, Y., Wu, G. M., Anafi, D. et al. (1999). Both familial Parkinson's disease mutations accelerate $\alpha$-synuclein aggregation. J. Biol. Chem. 274, 9843-9846.

11. Conway, K. A., Lee, S. J., Rochet, J. C., Ding, T. T., Williamson, R. E. \& Lansbury, P. T. (2000). Acceleration of oligomerization, not fibrillization, is a shared property of both $\alpha$-synuclein mutations linked to early-onset Parkinson's disease: implications for pathogenesis and therapy. Proc. Natl Acad. Sci. USA. 97, 571-576.

12. Serpell, L. C., Berriman, J., Jakes, R., Goedert, M. \& Crowther, R. A. (2000). Fiber diffraction of synthetic $\alpha$-synuclein filaments shows amyloid-like cross-beta conformation. Proc. Natl Acad. Sci. USA. 97, 4897-4902.

13. Conway, K. A., Harper, J. D. \& Lansbury, P. T. (2000). Fibrils formed in vitro from $\alpha$-synuclein and two mutant forms linked to Parkinson's disease are typical amyloid. Biochemistry, 39, 2552-2563.

14. Bussell, R. \& Eliezer, D. (2001). Residual structure and dynamics in Parkinson's disease-associated mutants of $\alpha$-synuclein. J. Biol. Chem. 276, 45996-46003.

15. Li, J., Uversky, V. N. \& Fink, A. L. (2001). Effect of familial Parkinson's disease point mutations A30P and A53T on the structural properties, aggregation, and fibrillation of human alpha-synuclein. Biochemistry, 40, 11604-11613

16. Choi, W., Zibaee, S., Jakes, R., Serpell, L. C., Davletov, B., Crowther, R. A. \& Goedert, M. (2004). Mutation E46K increases phospholipid binding and assembly into filaments of human $\alpha$-synuclein. FEBS Lett. 576, 363-368.

17. Hoyer, W., Cherny, D., Subramaniam, V. \& Jovin, T. M. (2004). Impact of the acidic C-terminal region comprising amino acids $109-140$ on $\alpha$-synuclein aggregation in vitro. Biochemistry, 43, 16233-16242.

18. Greenbaum, E. A., Graves, C. L., Mishizen-Eberz, A. J., Lupoli, M. A., Lynch, D. R., Englander, S. W. et al. (2005). The E46K mutation in $\alpha$-synuclein increases amyloid fibril formation. J. Biol. Chem. 280, 7800-7807.

19. van Raaij, M. E., Segers-Nolten, I. M. J. \& Subramaniam, V. (2006). Quantitative morphological analysis reveals ultrastructural diversity of amyloid fibrils from alphasynuclein mutants. Biophys. J. 91, L96-L98.

20. Fredenburg, R. A., Rospigliosi, C., Meray, R. K., Kessler, J. C., Lashuel, H. A., Eliezer, D. \& Lansbury, P. T. (2007). The impact of the E46K mutation on the properties of $\alpha$-synuclein in its monomeric and oligomeric states. Biochemistry, 46, 7107-7118.

21. Bertoncini, C. W., Fernandez, C. O., Griesinger, C., Jovin, T. M. \& Zweckstetter, M. (2005). Familial mutants of $\alpha-$ synuclein with increased neurotoxicity have a destabilized conformation. J. Biol. Chem. 280, 30649-30652.

22. Hoyer, W., Antony, T., Cherny, D., Heim, G., Jovin, T. M. \& Subramaniam, V. (2002). Dependence of $\alpha$-synuclein aggregate morphology on solution conditions. J. Mol. Biol. 322, 383-393.

23. Heise, H., Hoyer, W., Becker, S., Andronesi, O. C., Riedel, D. \& Baldus, M. (2005). Molecular-level secondary structure, polymorphism, and dynamics of full-length $\alpha$-synuclein fibrils studied by solid-state NMR. Proc. Natl Acad. Sci. USA. 102, 15871-15876.

24. Tycko, R. (2006). Molecular structure of amyloid fibrils: insights from solid-state NMR. Q. Rev. Biophys. 39, 1-55.

25. Heise, H. (2008). Solid-state NMR spectroscopy of amyloid proteins. ChemBioChem, 9, 179-189.

26. Kloepper, K. D., Zhou, D. H., Li, Y., Winter, K. A., George, J. M. \& Rienstra, C. M. (2007). Temperaturedependent sensitivity enhancement of solid-state NMR spectra of $\alpha$-synuclein fibrils. J. Biomol. NMR, 39, 197-211.

27. Kloepper, K. D., Hartman, K. L., Ladror, D. T. \& Rienstra, C. M. (2007). Solid-state NMR spectroscopy reveals that water is nonessential to the core structure of $\alpha$-synuclein fibrils. J. Phys. Chem. B, 111, 13353-13356.

28. Kloepper, K. D., Woods, W. S., Winter, K. A., George, J. M. \& Rienstra, C. M. (2006). Preparation of $\alpha$-synuclein fibrils for solid-state NMR: expression, purification, and incubation of wild-type and mutant forms. Protein Expression Purif. 48, 112-117.

29. Fernandez, C. O., Hoyer, W., Zweckstetter, M., JaresErijman, E. A., Subramaniam, V., Griesinger, C. \& Jovin, T. M. (2004). NMR of alpha-synuclein-polyamine complexes elucidates the mechanism and kinetics of induced aggregation. EMBO J. 23, 2039-2046. 
30. Seidel, K., Lange, A., Becker, S., Hughes, C. E Heise, H. \& Baldus, M. (2004). Protein solid-state NMR resonance assignments from (C-13, C-13) correlation spectroscopy. Phys. Chem. Chem. Phys. 6, 5090-5093.

31. Pauli, J., Baldus, M., van Rossum, B., de Groot, H. \& Oschkinat, H. (2001). Backbone and side-chain ${ }^{13} \mathrm{C}$ and ${ }^{15} \mathrm{~N}$ signal assignments of the alpha-spectrin $\mathrm{SH} 3$ domain by magic angle spinning solid-state NMR at 17.6 Tesla. ChemBioChem, 2, 272-281.

32. Ritter, C., Maddelein, M.-L., Siemer, A. B., Lührs, T., Ernst, M., Meier, B. H. et al. (2005). Correlation of structural elements and infectivity of the HET-s prion. Nature, 435, 844-848.

33. Andronesi, O. C., Becker, S., Seidel, K., Heise, H., Young, H. S. \& Baldus, M. (2005). Determination of membrane protein structure and dynamics by magicangle-spinning solid-state NMR spectroscopy. J. Am. Chem. Soc. 127, 12965-12974.

34. Siemer, A. B., Arnold, A. A., Ritter, C., Westfeld, T., Ernst, M., Riek, R. \& Meier, B. H. (2006). Observation of highly flexible residues in amyloid fibrils of the HET-s prion. J. Am. Chem. Soc. 128, 13224-13228.
35. Heise, H., Köhler, F. H. \& Xie, X. L. (2001). Solidstate NMR spectroscopy of paramagnetic metallocenes. J. Magn. Reson. 150, 198-206.

36. Der-Sarkissian, A., Jao, C. C., Chen, J. \& Langen, R. (2003). Structural organization of $\alpha$-synuclein fibrils studied by site-directed spin labeling. J. Biol. Chem. 278, 37530-37535.

37. Miake, H., Mizusawa, H., Iwatsubo, T. \& Hasegawa, M. (2002). Biochemical characterization of the core structure of alpha-synuclein filaments. J. Biol. Chem. 277, 19213-19219.

38. Del Mar, C., Greenbaum, E. A., Mayne, L., Englander, S. W. \& Woods, V. L., Jr (2005). Structure and properties of $\alpha$-synuclein and other amyloids determined at the amino acid level. Proc. Natl Acad. Sci. USA. 102, 15477-15482.

39. Cornilescu, G., Delaglio, F. \& Bax, A. (1999). Protein backbone angle restraints from searching a database for chemical shift and sequence homology. J. Biomol. NMR, 13, 289-302.

40. Fung, B. M., Khitrin, A. K. \& Ermolaev, K. (2000). An improved broadband decoupling sequence for liquid crystals and solids. J. Magn. Reson. 142, 97-101. 